\title{
EL PAPEL QUE DESEMPEÑAN LAS TEORÍAS EN LA BIOLOGÍA
}

\author{
BARBERÁ, $O$. \\ Departament de Didàctica de les Ciències Experimentals. Universitat de València. Apartado de Correos 22045. \\ 46071 València.
}

\begin{abstract}
SUMMARY
This paper tries to emphasize the importance of the term theory interpretation and its misuse in science education. It also discusses the convenience of teaching biology as a hypothetic-deductive science, and the role that conceptual mapping plays on these items.
\end{abstract}

\section{INTRODUCCIÓN}

La mayoría de los avances científicos, tanto los considerados grandes como los menores, proceden de la enunciación, desarrollo y aplicación de teorías; por ello el estudio de las teorías debe de ser un objetivo principal en la educación científica (Hodson 1986).

En la realidad, la representación de las teorías que se encuentra en los libros de texto de biología es escasa (Hill 1986, Lerner y Bennetta 1988, Lewis 1988). Durante el siglo pasado y la primera mitad del presente, la biología ha venido siendo definida como una ciencia descriptiva. A esto ha contribuido, sin duda, la gran cantidad de trabajo que durante este periodo se realizó sobre taxonomía, morfología y estudio natural. Pero durante ese mismo periodo, e inclusive muy anteriormente, la biología ya era una ciencia con carácter hipotéticodeductivo, lo que hoy denominamos biología teórica.

Data de 1628 la primera noticia que tenemos de un sistema de razonamiento hipotético-deductivo en ciencias naturales: la teoría de William Harvey sobre la circulación de la sangre, expresada en su obra Exercitatio anatomica de motu cordis et sanguinis in animalibus (Laín Entraigo 1957, López Piñero 1973). En el siglo XIX, son muy numerosas las teorías formuladas con estas características en campos como la fisiología, la evolución, la reproducción, la herencia, etc. Ya en nuestro siglo, han sido muy fructíferas las concernientes a la estructura y función del
ADN, a la acción entre antígenos y anticuerpos, y otras muchas que todos tenemos en mente. ¿Cuál es entonces la razón de que aún se presente la biología como una ciencia eminentemente descriptiva?

\section{ACEPCIONES DEL VOCABLO TEORÍA}

Seguramente son variadas y numerosas las razones que explican este fenómeno, pero únicamente desarrollaré la que, a mi juicio, es la principal responsable: Ia mayoría de libros de texto y una proporción no despreciable de los docentes de los distintos niveles de enseñanza siguen utilizando el método descrito por Francis Bacon como el paradigma de método científico (Hodson 1988), a saber: recolección de datos, clasificación de éstos y, si han sido correctamente realizados los dos primeros pasos, entonces todoello cristalizará en una Ley General que la Naturaleza deberá cumplir (Fig. 1). Esta forma de presentar el método científico como único y en una forma puramente inductiva (Hodson 1982) conduce a un error en la metodologíacientífica (error que incluso Darwin cometió, aunque de forma puramente dialéctica, véase Gould 1986); este hecho, unido a las confusas definiciones que existen sobre lo que es una teoría, hace que éstas no queden justamente representadas en el cuerpo de conocimiento de la biología. 
Figura I

Esquena de los métodos inductivo e hipotético-deductivo. EI método inductivo es defectuoso en varios niveles: 1) la decisión de recoger algún tipo de información precisa de una teoría preexistente,

2) una afirmación general nunca puede derivarse lógicamente a partir de un número limitado de observaciones, etc. En el hipotéticodeductivo la falsación también presenta dificultades filosoficas (según Hill 1985).

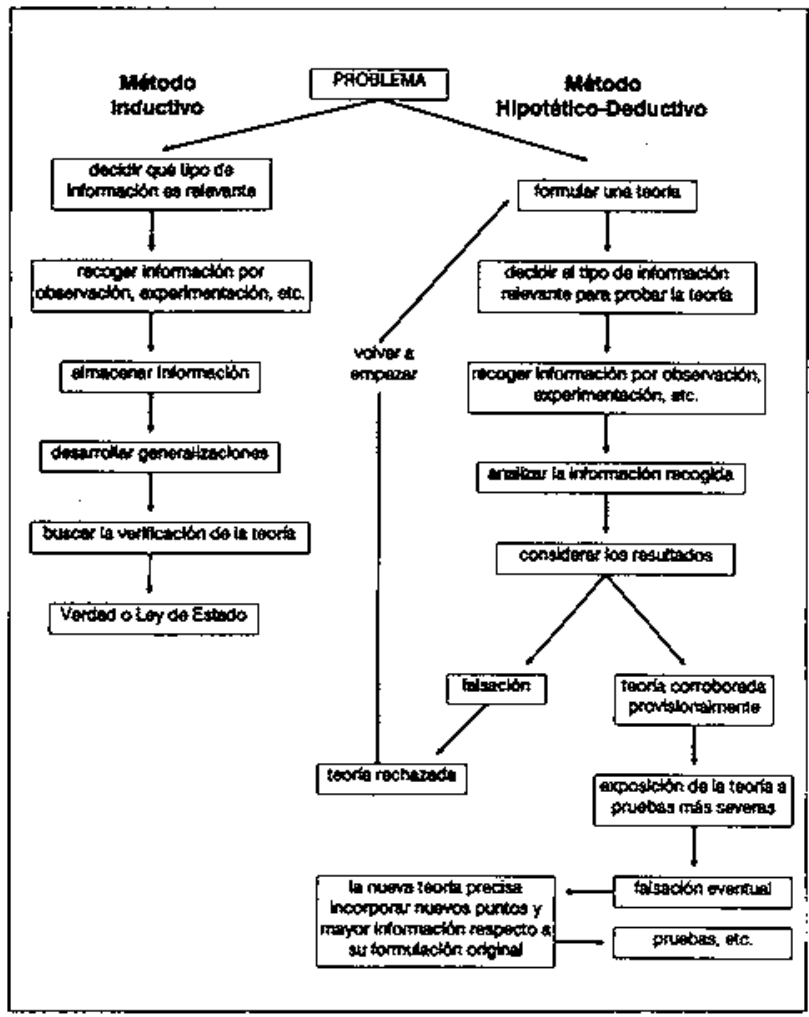

En el diccionario de la Real Academia Española (1970) podemos leer:

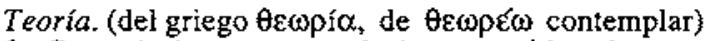
f. Conocimiento especulativo considerado con independencia de toda aplicación. 2. Serie de las leyes que sirven para relacionar determinado orden de fenómenos. 3. Hiporesis cuyas consecuencias se aplican a toda una ciencia o a parte muy importante de la misma. 4. Procesión religiosa entre los antiguos griegos.

Julio Casares, en su diccionario ideológico de la Lengua Española (Casares 1989), añade una acepción más a las reseñadas arriba: «Ley o sistema de leyes que se đeducen de Ia observación de ciertos fenómenos científicos, y sirven para relacionarlos y explicarlos». A pesar del uso de la palabra deducir, el proceso mental que representa esta acepción de teoría es claramente inductivo.

Cuando se habla de teorías en ciencias, estas definiciones que nos aportan los diccionarios de español no hacen sino confundir. Además, parece ser que otros países con una mayor tradición en epistemología y en filosofía de la ciencia adolecen de los mismos problemas en cuanto a
Ias definiciones de este vocablo (Futuyma 1988). El mal uso del término teoría es muy común, y esto puede llevar a confusión a muchos estudiantes.

Una teoría es algo más que una simple inducción o una determinada idea que se supone que representa la realidad. Es un modelo quasi-geométrico de razonamiento, cuyo contenido es un conjunto de ideas formuladas como postulados o premisas básicas, unas líneas de razonamiento, algunos hechos y algunas definiciones. Las líneas de razonamiento son las encargadas de construir la estructura, $y$ lo pueden hacer de tres formas: 1) utilizando los hechos para corroborar los correspondientes postulados, 2) utilizando los hechos y los postulados para explicar otros hechos relacionados, o 3 ) utilizando los postulados y los hechos para predecir posibles nuevos hechos (Fig. 2) .

Figura 2

Esquema de una teoría: $\mathrm{P}=$ postulado; $\mathrm{R}=$ línea de razonamiento; $\mathrm{H}$ $=$ hecho; $\mathrm{Pt}=$ predicción (según Holton, 1979).

$p$
$p$
$p$
$p$
$P$
$p$<smiles>[R][R]([R])[H]</smiles>

Una teoria es un constructo intelectual que intenta describir una realidad observada y proporcionar modelos aproximados y útiles (véase Hođson 1988, para una clarificación entre Ios conceptos de teoría y modelo). Las teorías no son descubiertas, sino inventadas, y todas ellas son susceptibles de modificación al aparecer algunos hechos que sean inconsistentes con la teoría propuesta. Si la inconsistencia es muy profunda puede llegarse incluso al abandono de la propia teoría, sea o no reemplazada por otra.

Unas cuantas teorías que en un determinado momento tuvieron una amplia aceptación por parte de la comunidad científica han sido actualmente descartadas, pero no es éste el destino de la mayoría; por el contrario, muchas se han imbricado totalmente en el cuerpo del conocimiento, y sus postulados han obtenido un soporte tan fuerte que han llegado a ser aceptadas como hechos generales o leyes. Es lo que el profesor R.W. Lewis Ilama embedded theories; podría traducirse como teorías empotradas en 
ese cuerpo de conocimiento al que antes hacía alusión. El mismo autor también nos proporciona unos cuantos ejemplos de ellas (Lewis 1982, 1986 y 1988); así, la teoría microbiana de la enfermedad, tan brillantemente propuesta por Louis Pasteur y Robert Koch en el último cuarto del pasado siglo (Dubos 1985), ha llegado hasta nuestros dias en forma de hecho general: una enfermedad es causada por microorganismos que se desarrollan y multiplican en el huésped.

Estas teorías ampliamente aceptadas han sido tan sumamente útiles a la hora de explicar hechos y realizar predicciones, que, incluso después de demostrar deficiencias, no han sido descartadas. Por ejemplo, Ia teoría microbiana falla cuando se trata de enfermedades debidas a deficiencias, como la enfermedad del legionario, las avitaminosis, el síndrome de inmunodeficiencia adquirida o algunas hereditarias. En lugar de descartarlas, reconocemos sus limitaciones, sus fronteras en el rango de aplicabilidad.

\section{CONSECUENCIAS DIDÁCTICAS}

Estas teorías fuertemente establecidas son las que proporcionan estructura lógica a las disciplinas cientifficas, pero no suelen presentarse como teorías en los libros y actos docentes, y tampoco se les proporciona estructura de teoría al enunciarlas, lo que oculta el carácter de la ciencia y dificulta la tarea de los aprendices.

EI uso de mapas conceptuales en Ias aulas (Novak 1980 y 1988) puede ser un medio importante para corregir esta situación. La confección de estos mapas solicita del estudiante la construcción de relaciones lógicas entre hechos, conceptos y actividades, lo que le obliga a realizar conexiones entre todo aquello que en los libros se presenta como secuencias lineales. Si lo que se pretende en la enseñanza es algo más que aprender maquinalmente una secuencia lineal, los mapas conceptuales deben ser un instrumento imprescindible en la enseñanza de las ciencias.

Si aceptamos que la formulación, comprobación y aplicación de teorías es la actividad central en la construcción del conocimientocientífico, que las teorías de amplia aceptación son las que proporcionan la estructura lógica a sus distintas disciplinas, y que la estructura teórica proporciona al estudiante la forma más sencilla de construir modelos lógicos de asociación entre conceptos, la educación científica que no presente las teorías como tales, no será útil para transmitir la esencia científica y perderá la oportunidad de presentar a la ciencia en su más accesible formato.

La educación en biología está actualmente repieta de dogmatismo, y una buena forma de intentar erradicarlo de una vez por todas, de las aulas, es poner en práctica la enseñanza del verdadero carácter de la biología; hay que reexaminar los contenidos preguntándose cuáles son las teorías que van a ser enseñadas a to largo del curso, identificándolas de entre las secuencias lineales de lo que es presentado como hechos, buscando sus postulados, proporcionando ejemplos de líneas de razonamiento útiles para corroborarlas, para explicar fenómenos y realizar predicciones, $y$, por supuesto, delimitando su rango de aplicabilidad. Esta práctica puede acabar definitivamente con la visión de que la biología es una ciencia que acumula los hechos más rápidamente que las teorias capaces de acomodarlos (Ost 1987).

La enseñanza de una teoría en un formato adecuadamente estructurado es sencilla. Proporcionemos al discente el conjunto de postulados para empezar. No caigamos en el error de querer que el aprendiz derive correctamente los postulados a partir de los hechos disponibles que haya aprendido anteriormente. La formulación de un juego de postulados útil es una tarea harto dificultosa, que normalmente ha sido llevada a cabo por personas creativas y con un altísimo nivel de información acerca del fenómeno en cuestión. Si los profesores no cumplimos normalmente estas premisas, no pidamos a los alumnos que Ileven a cabo tareas que se pueden considerar a priori como imposibles.

Una vez los estudiantes poseen un conjunto de hechos y un juego de postulados accesible, es cuando las cuestiones que vaya planteando el docente deben ir dirigidas hacia cómo explicar los hechos a partir de los postulados, cómo validar o refutar las distintas premisas, cómo predecir nuevas situaciones, qué líneas de razonamiento se exponen para responder estas preguntas, etc. Si eI profesor conoce a fondo la estructura general de la teoráa, no le será difícil conseguir un conjunto apropiado de preguntas para cada situación de clase.

Para ilustrar esta técnica, proporcionaré un ejemplo en el que se hace uso de los mapas conceptuales para trabajar las líneas de razonamiento de una teoría con los alumnos. He elegido las dos teorias principales enunciadas por Darwin (1859) en su Origen de las especies: la teoría de la descendencia con modificación y la teoría de la selección natural. Una vez poseen los estudiantes los juegos de postulados de ambas (éstos han sido descritos en varios trabajos; Lewis 1980 y 1986), se puede sacar a relucir una de las críticas más comunes en contra de las tesis darwinistas: el argumento del diseño (Dawkins 1988). Este argumento, que ha venido siendo utilizado desde la edición de El Origen, consiste principalmente en el sentimiento de admiración que nos produce la observación de un objeto con un mecanismo muy complejo, y la subsiguiente implicación de que resulta evidente que algo tan maravilloso y tan bien diseñado no puede haber sido construido por el azar. El ejemplo histórico de este tipo de objetos, que Darwin denominó córganos de extrema perfección y complejidad», es el ojo.

Obviamente, el orden natural no puede ser fruto exclusivo del azar, y Darwin compartía esta opinión. Ésa fue la razón que le Ilevó a elaborar su teoría explicativa en dos partes (dos subteorías o principios), permitiendo que en la primera prevaleciera el azar, pero excluyéndolo estrictamente de la segunda (actualmente se comienza a reconocer que esta exclusión quizá no sea tan estricta, y el azar juega algún papel determinista en la historia de la vida; vêase Gould 1984). Según Darwin, en las poblaciones se desarrolla una gran cantidad de variación heredable, 
Figura 3

Esquema conceptual en el que se hace patente el error en que incurre el argumento del diseño, una de las más corrientes críticas a las posturas darwinistas: se puede observar la desviación que se produce del sujeto principal de la crítica, el azar, desde conceptos de la teoría de selección natural hacia la de descendencia con modificación, en un intento de falsar las pruebas presentadas sobre el mecanismo de selección natural. Los casos del ojo, la adaptación al vuelo y la aparición de la célula eucariota son los más representativos de ese argumento del diseño.

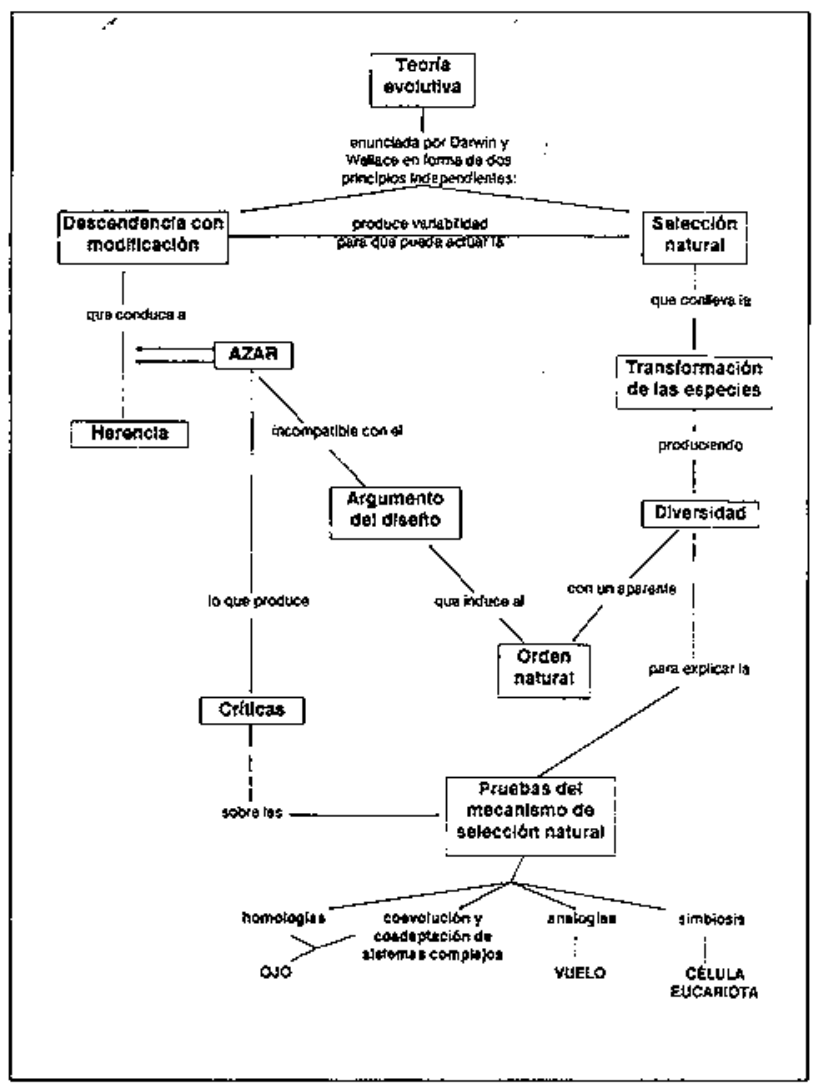

que es la que suministra la materia prima para la posterior acción directora de la selección natural. Darwin consideró el almacenamiento de variaciones como aleatorio respecto a la dirección del cambio evolutivo, si bien nunca gozó de una teoría consistente para explicar la aparición y heredabilidad de esas variaciones (Castrodeza 1988).

Darwin se expresó con una claridad admirable (Korey 1987) y, a pesar de ello, algunos críticos llevan más de un siglo malinterpretando este punto fundamental de su teoría. Este argumento del diseño basa su crítica a las tesis darwinistas en un punto erróneo: la confusión de la selección natural con el azar. En el mapa conceptual de la figura 3 se puede observar cómo se produce una desviación del objeto de crítica desde una subteoría a la otra, en la que es empleado erróneamente.

\section{CONCLUSIONES}

Estos modelos de pensamiento racional no sólo se presentan en la enseñanza de la biología y del resto de ciencias, sino que son imprescindibles en el mundo intelectual, y de mucha utilidad en el mundo práctico. Casi toda actividad humana se desenvuelve alrededor de juegos de ideas de reducido número. Aquéllos que desconozcan este hecho sobre el pensamiento racional y que no to pongan conscientemente en práctica, partirán con sus facultades mermadas para acercarse al mundo científico antes de comenzar la hazaña. J. Maynard-Smith, uno de los más destacados teóricos de la biología, identificó la dificultad de aprender biología con la presencia en ella de «un montón de maIditos hechos» (Hit1 1986). Estoy seguro de que este punto de vista debe ser similar al de muchos estudiantes, pero también me atrevo a asegurar que todos los que lo comparten han carecido en su educación científica en biología de los modelos y procesos sobre los que esta ciencia está constituida.

Naturalmente, un curso de biología consiste en algo más que una lista de teorías, pero no es éste el objeto que me habia propuesto al escribir estas líneas. Tampoco me gustaría que quedase en el lector la impresión de menosprecio por mi parte hacia algunas áreas de conocimiento de esta ciencia. El presentar el carácter de la biología como hipotético-deductivo no implica en absoluto que la biología descriptiva deje de ser una parte importante. El mismo Maynard-Smith, al hablar sobre la piedra angular de la biología, lo ha expresado de la siguiente forma: «nuestro conocimiento de la evolución depende đe una combinación de teorías correctamente formuladas y de un extenso conocimiento comparativo" (Lewis 1988). Este conocimiento comparativoes, a menudo, fundamentalmente descriptivo.

No obstante, tópicos como pueden ser la taxonomía, la morfología o el estudio natural, considerados tradicionalmente descriptivos en la biologia, se enmarcan actualmente en un modelo hipotético-deductivo a través de la ecología, y prácticamente no queda ninguna parte que sea tratada de manera exclusivamente descriptiva.

Si la estructura de la biología es un entramado de teorías, su enseñanza debe ser abordada desde esa red, y no únicamente desde los hechos; para esto es necesaria una clara identificación de las teorías, condición que no suele darse ni en los tex tos ni en los procesos de enseñanzaaprendizaje que habitualmente practicamos sobre esta disciplina. 


\section{REFERENCIAS BIBLIOGRÁFICAS}

CASARES, J. 1989. Diccionario ideológico de la Lengua Española. (Gustavo Gili: Barcelona).

CASTRODEZA, C., 1988. Teoria historica de la Selección Natural. (Alhambra: Madrid).

DARWIN, C., 1859.The Origin of Species. (Penguin: Londres). Traducción española: El origen de las especies, 1983.(Serbal: Barcelona).

DAWKINS, R, 1988. El relojero ciego. (Labor: Barcelona).

DUBOS, R.J., 1985. Pasteur. (Salvat ed.: Barcelona).

FUTUYMA, D.J., 1988. Evolutionary biology, 2nd. ed. (Sinauer Associates: Sunderlan, MA, EUA).

GOULD, S.J., 1984. Las riquezas de la fortuna, en: Dientes de gallina y dedos de caballo. (Hermann Blume: Barcelona).

GOULD, S.I., 1986. El camino de enmedio de Darwin, en: El pulgar del panda. (Orbis: Barcelonit).

HARVEY, W., 1628. Exercitatio anatomica de motu cordis et sanguinis in animalibus. (Frankfurt am Main).

HILL, L., 1985. Bioiogy, philosophy and scientific method, Journal of Biological Education, 19, pp. 227-231.

HILL, L., 1986. Teaching and the theory and practice of biology, Journal of Biological Education, 20, pp. 112-116.

HODSON, D., 1982. Is there a scientific method?, Journal of Chemical Education, 19, pp. 112-115.

HODSON, D., 1988. Filosofía de la ciencia y educación científica, en: Constructivismo y enseñanza de las ciencias, (Diada ed.: Sevilia).
HOLTON, G., 1979. Constructing a theory: Einstein's model, American Scholar, 48, pp. 309-340.

KOREY, K., 1987. Charles Darwin. Textos Fundamentales. (Paidós Ibérica: Barcelona).

LAÍN ENTRALGO, P., 1957. Harvey in the history of scientific thought, Journal of Hystory of Medicine, 12, pp. 220-231.

LERNER, L.S. y BENNETTA, W.J., 1988. The treatment of theory in textbooks, The Science Teacher, 55, pp. 37-41.

LEWIS, R.W., 1980. Evolution: asystem of theories, Perspectives in Biology and Medicine, 23, pp. 551-572.

LEWIS, R.W., 1982. Theories, structure, teaching and leaming, BioScience, 32, pp. 734-737.

LEWIS, R.W., 1986. Teaching the theories of evolution, The American Biology Teacher, 48, pp. 334-347.

LEWIS, R.W., 1988. Biology: a hypothetico-deductive science, The American Biology Teacher, 50, pp. 362-366.

LOPEZPIÑERO, J.M., 1973. Harvey's doctrine of the circulation of the blood in seventeenth-century in Spain, Journal of Hystory of Medicine, 28, pp. 230-242.

NOVAK, J.D., 1980. Leaming theory applied to the biology classroom, The American Biology Teacher, 42, pp. 280-285.

NOVAK, J.D., 1988. Constructivismo humano: un consenso emergente, Enseñanza de las Ciencias, 6, pp. 213-223.

OST, D.H., 1987. The evolution of a biology curricutum, The American Biology Teacher, 49, pp. 153-156.

REAL ACADEMIA ESPAÑOLA, 1970. Diccionario de la Lengua Española. (Madrid). 